\title{
An Improved White Space Prediction Algorithm for Cognitive Radio Systems
}

\author{
Nguyen Huu Phong1, Vo Nguyen Quoc Bao ${ }^{2}$, Huynh Huu Tue ${ }^{3}$ \\ ${ }^{1}$ Broadcast Research and Application Center, Ho Chi Minh City, Vietnam \\ ${ }^{2}$ School of Telecommunications, Posts and Telecommunications Institute of Technology, Ho Chi Minh \\ City, Vietnam \\ ${ }^{3}$ International University, Ho Chi Minh City, Vietnam
}

Correspondence: Vo Nguyen Quoc Bao, baovnq@ptithcm.edu.vn

Manuscript communication: received 27 November 2013, accepted 9 January 2014

\begin{abstract}
Cognitive radio (CR) is a promising technology to enhance the current low usage of limited frequency resources. TV white space (TVWS) - TV bands at a particular time in a particular geographic area that are not being used by licensed services - is perceived as the most suitable frequency bands for CR. This paper proposes a new prediction TVWS algorithm for CR systems based on the ITU 1546.1 and the Okumura-Hata models. The proposed algorithm is verified with the data of 22 provinces in the South of Vietnam. The numerical results confirm the advantage of the proposed algorithm as well as the possibility of TVWS CR networks.
\end{abstract}

Keywords- TV White Space, Cognitive Radio, ITU-1546.1, Okumura-Hata

\section{INTRODUCTION}

Currently, spectrum allocated to Television (TV) broadcasting in the very high frequency (VHF), $54-216 \mathrm{MHz}$, and ultrahigh frequency (UHF), $470-698 \mathrm{MHz}$, is very attractive for secondary wireless applications. It is mainly due to the excellent signal propagation characteristics for the coverage of large areas [1]. Some recent spectrum measurements, e.g. [2-5] , indicate that most of the allocated spectrum in general and the TV bands in particular are underutilized.

TV signals are usually broadcast at high and very high transmit powers. To avoid co-channel interference, TV networks operating at the same frequencies are usually separated by significant distances while neighboring TV networks use different frequency bands. As a result, spectral remnants among them exist, which can be used locally for low-power applications without disturbing TV reception. In particular, TVWS refers to the TV bands at a particular time in a specific geographic area that are not being used by licensed services [6].

In many regions, Internet access is limited and/or costly due to the roll-out of fixed network infrastructure. Wireless systems making use of TV white spaces, i.e. IEEE 802.22 wireless regional area networks (WRAN), is a promising solution since it can offer an inexpensive alternative for Internet covering the last mile of rural communities. TVWS systems are able to detect available TV channels using spectrum sensing technique, also known as cognitive radio (CR) technology [7]. TVWS devices operate on the VHF/UHF band, i.e. $54-862 \mathrm{MHz}$, where television services exist, but do not cause interference to these TV systems. Base stations of TVWS networks use low power output, i.e. $4 \mathrm{~W}$ EIRP in the U.S., being capable of covering a radius of about $17-30 \mathrm{~km}$, depending on antenna height and power output. As a result, wireless systems use of TVWS is suitable for the area rural demand for multimedia communication and broadband services.

An important issue for TVWS networks is to protect primary TV networks including analog TV, DVB-T and DVB-T2. Many recent studies, e.g. see [8-11], showed that white space devices (WSD) can simultaneously operate with licensed networks thanks to power adaptation algorithms. In [8], the problem of allocating transmit power was investigated. For the interference constraint to primary users, the optimization problem of maximizing total system up-link throughput has been solved by the water filling algorithm. In [9], a novel methodology for calculating the maximum permitted WSD EIRP subject to a target degradation in location probability was proposed. In [10], an estimation model of the available digital TV channels for CR operations was proposed along with the concept of keep away region. Based on the proposed model, a comprehensive and profound analysis on the available spectrum for $\mathrm{CR}$ devices for 18 cities in the UK has been shown. In [11], a novel power control algorithm based on the maximum allowed transmission power was proposed. Numerical results show that the proposed algorithm outperforms the traditional keep-away region algorithm in terms of numbers of vacant channels. However, the estimation of the received TV signal power and the number of 
available TV channels via computer simulations heavily depend on the choice of the radio propagation model.

The contribution of this paper has two parts: i) to select the best radio propagation models and ii) to propose a new algorithm to calculate TVWS channels available for TVWS networks. The algorithm also allows to take into account specific conditions in Vietnam. Field measurements were conducted to validate the proposed algorithm as well as to confirm its advantages.

The structure of this paper is as follows. Section 2 presents the 802.22 network deployment. Television protected contours are presented in Section 3, where two path-loss models including ITU-R 1546.1 and FCCdefined statistical propagation model are shown and then verified with the measurement results. In Section 4, we consider three channel models between TV receivers and WSDs. The measurement results confirm that the Okumura-Hata is the best model. Based on the two above-selected channel models, i.e. ITU 1564.1 and Okumura-Hata, we propose a white space prediction algorithm for TVWS radio systems. Finally, Section 7 provides closing remarks.

\section{System Model}

An envisioned deployment configuration for IEEE 802.22 wireless regional area network using white spaces in the television (TV) frequency spectrum is shown in Figure 1. IEEE 802.22 WRANs are designed to operate in the TV broadcast bands while assuring that no harmful interference is caused to the incumbent operation: digital TV and analog TV broadcasting, and low power licensed devices [12]. In a WRAN cell, the network typically consists of a base station (BS) and a number of customer premise equipments (CPE) ${ }^{1}$. In this paper, we limit our study to fixed CPEs. The maximum transmit EIRP is of $4 \mathrm{~W}(36 \mathrm{dBm})$ based on a conducted power of $1 \mathrm{~W}$ and a CPE antenna gain of $6 \mathrm{dBi}^{2}$.

The IEEE 802.22 standard prohibits both WRAN BS and CPE operating in the co-channel and the first adjacent channel within the protected noise-limited contour Furthermore, the CPE antenna should be mounted $10 \mathrm{~m}$ above the ground and at least separated by $10 \mathrm{~m}$ from the closest TV receiver antenna [12]. Stated another way, if a TV station is operating over channel $N$ in the UHF band, no communications of BS or CPE inside the Grade B contour are allowed. Moreover, a keep out distance is required between a BS and a TV receiver if the BS is located outside the Grade B contour and is operating on channel $N$ or $N+1$. For channel $N \pm i$ with $i \geq 2$, the communication for WRAN BS and CPE are possible subject to limit their EIRP below certain levels to avoid interference to nearby TV receivers. It is obvious that this distance is a function of the maximum allowable effective isotropic radiated power (EIRP) of the WRAN

\footnotetext{
${ }^{1}$ Portable devices are not available at this moment for testing and measurement.

${ }^{2}$ Other combinations are also possible as long as the maximum transmit EIRP of $4 \mathrm{~W}$ is not exceeded
}

BS, the operation mode (fixed or personal/portable mode), and the transmit antenna height of WSDs. As a result, the geographical characteristics, local shape of each area, and radio propagation environments should be taken into account when determining the keep out distance.

\section{Television Protected Contours}

The prediction of the actual TV white space usability requires an accurate estimation of the broadcast coverage areas. A TV station service coverage depends on the transmission effective radiated power (ERP), transmit antenna height above average terrain (HAAT) and operating frequency band. In the US and Canada, DTV service is available in the area within the grade B contour, where the field strength values are $28 \mathrm{dBu}$ for low VHF, $36 \mathrm{dBu}$ for high VHF, and $41-20 \log [615 /$ channel mid-frequency in MHz] dBu for UHF [13].

Currently, Vietnam has adopted PAL D/K for analogue TV transmission and DVB T/T2 for digital TV transmission. For PAL D/K, the median field strength in Bands I, III, IV or V for which protection against interference is planned should not be lower than values given in Table $\mathrm{I}^{3}$ [14]. Note that these values refer to the field strength at a height of $10 \mathrm{~m}$ above ground level.

Table I

The Median Field Strength for Analogue Terrestrial TELEVISION

\begin{tabular}{|l|c|}
\hline Band & $\mathrm{dBuV} / \mathrm{m}$ \\
\hline \hline I (41-68 MHz) & 48 \\
\hline III (162-230 MHz) & 55 \\
\hline IV (470-582 MHz) & 65 \\
\hline V (582-960 MHz) & 70 \\
\hline
\end{tabular}

For DVB-T and DVB-T2, the median field strengths are given respectively in Table II [15] and Table III [16].

Table II

The Median Field Strength for DVB-T

\begin{tabular}{|l|c|}
\hline Band & $\mathrm{dBuV} / \mathrm{m}$ \\
\hline \hline Band III VHF (European Union) & 42 \\
\hline Band IV/V UHF (European Union) & 47 \\
\hline Location probability & $70 \%$ \\
\hline$C / N$ in dB & 20 \\
\hline
\end{tabular}

Table III

The Median Field Strength for DVB-T2

\begin{tabular}{|l|c|}
\hline Band & $\mathrm{dBuV} / \mathrm{m}$ \\
\hline \hline Band III VHF (European Union) & 47.4 \\
\hline Band IV /V UHF (European Union) & 54.3 \\
\hline Location probability & $95 \%$ \\
\hline$C / N$ in dB & 20 \\
\hline
\end{tabular}

To determine television protected contour, we next consider two models including ITU-R 1546.1 and FCCdefined statistical propagation model.

\footnotetext{
${ }^{3}$ The values shown for Bands IV and V should be increased by 2 $\mathrm{dB}$ for system $\mathrm{K}$.
} 


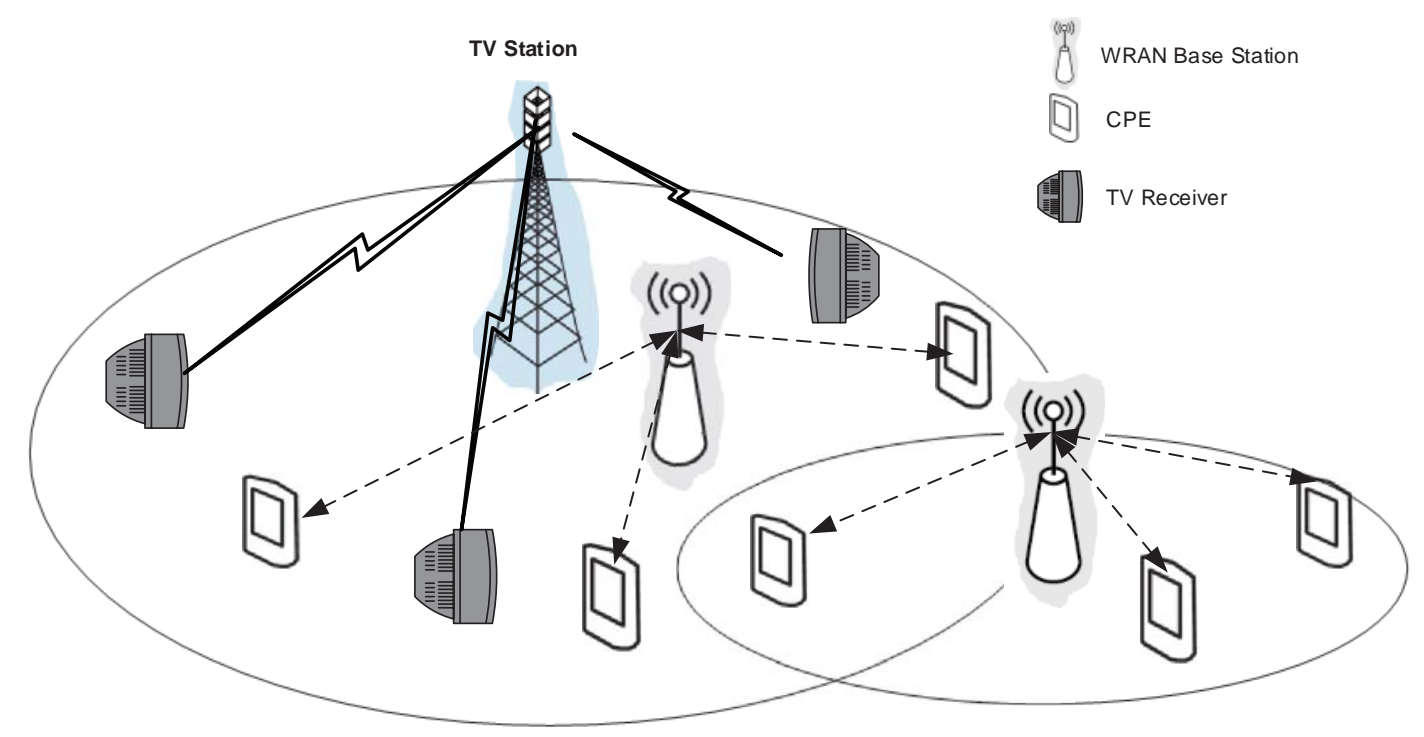

Figure 1. An example of 802.22 network deployment.

\subsection{ITU-R 1546.1}

Recommendation ITU-R 1546.1 is used as a method for point-to-area prediction of field strength for the broadcasting, land mobile, maritime mobile and certain fixed services (e.g. those employing point-to-multipoint systems) in the frequency range $30 \mathrm{MHz}$ to 3000 $\mathrm{MHz}$ and for the distance range $1 \mathrm{~km}$ to $1000 \mathrm{~km}$. Although field strengths may be read directly from the curves presented as figures in Annexes 2, 3 and 4 of [17], to assist in computer implementations, the stepby-step procedure should be proceeded as follows [17]:

- Step 1: For given transmit height, $h_{1}$, we calculate the dimensionless parameter $k$

$$
k=\log _{2}\left(\frac{h_{1}}{9.375}\right)
$$

where $k$ is an integer in the range 0 to 7 representing each member line of a family starting at $h_{1}=9.375 \mathrm{~m}$ and finishing at line $h_{1}=1200$ $\mathrm{m}$. For $h_{1}$ outside the range, i.e. $[9.375,1200]$, the extrapolation is needed.

- Step 2: Calculate an intermediate field strength, $E_{u}$, at distance $d$ as follows:

$$
E_{u}=p_{b} \log \left[\frac{10^{\frac{E_{1}+E_{2}}{p_{b}}}}{10^{\frac{E_{1}}{p_{b}}}+10^{\frac{E_{2}}{p_{b}}}}\right]
$$

where $p_{b}=d_{0}+d_{1} \sqrt{k}$ and

$$
\begin{aligned}
\mathrm{E}_{1}= & \left(a_{0} k^{2}+a_{1} k+a_{2}\right) \log d \\
& +0.1995 k^{2}+1.8671 k+a_{3}
\end{aligned}
$$

and $E_{2}=E_{r e f}+E_{o f f}$. In $E_{2}, E_{r e f}$ is of the form

$$
\begin{aligned}
E_{r e f}= & b_{0}\left[\exp \left(-b_{4} 10^{\xi}\right)-1\right] \\
& +b_{1} \exp \left[-\left(\frac{\log d-b_{2}}{b_{3}}\right)^{2}\right]-b_{6} \log d+b_{7}
\end{aligned}
$$

with $\xi=\log (d)^{b_{5}}$ and

$$
E_{o f f}=\frac{c_{0} k}{2}\left[1-\operatorname{tgh}\left[c_{1}\left(\log d-c_{2}-\frac{c_{3} k}{c_{4}}\right)\right]\right]+c_{5} k^{c_{6}} \text {. }
$$

Parameters $a_{k}, b_{k}, c_{k}$ and $d_{k}$ in (3), (4) and (5) are given in [17, Table 6] for all frequencies and time percentages of the land curves.

- Step 3 (Final): Calculate the field strength, $E_{b}$, as follows:

$$
E_{b}=p_{b b} \log \left[\frac{10^{\frac{E_{u}+E_{f s}}{p_{b b}}}}{10^{\frac{E_{u}}{p_{b b}}}+10^{\frac{E_{f s}}{p_{b b}}}}\right],
$$

where $E_{f s}$ is free-space field strength defined in Annex 5, which is

$$
E_{f_{S}}[d B \mu V / m]=106.9-20 \log d
$$

and $p_{b b}$ denotes the blend coefficient (usually $\left.p_{b b}=8\right)$. Field-strength values for an arbitrary frequency, percentage of time should be obtained by interpolating between the values for the nominal frequency values of 100, 600 and $2000 \mathrm{MHz}$.

\subsection{FCC-defined Statistical Propagation Model}

FCC has long defined protected service areas for TV transmitters, i.e. the Grade-B service contour for analog TV stations [13] or the noise limited contour (NLC) for digital TV stations. In particular, these areas define regions where incumbent TV receivers must be protected from harmful interference or TV signals are generally receivable with an outdoor TV antenna. To predict TV broadcast service areas, FCC-defined statistical propagation models, which are based on empirical measurements, are used. The $F(X, Y)$ model considers both a location and time-reliability variable, e.g. $\mathrm{X} \%$ locations and $Y \%$ of time, representing the statistical percentage of locations and time that service is available at least 
at the field strength level. The FCC also considers standard co-channel and adjacent channel interference tolerance levels for TV receivers. Such levels help ensure interference-free reception of TV services within their protected contours. More detail on the $\mathrm{F}(\mathrm{X}, \mathrm{Y})$ model can be found on [18].

\subsection{Comparison with the Measurement Results}

In this subsection, we compare the above-mentioned channel models with the measurement results performed at Ho Chi Minh City and Binh Duong province on March 2013. We consider two TV channels: i) VTV3 (channel 62 with frequency $802 \mathrm{MHz}$, transmit antenna height: $92.5 \mathrm{~m}$, receive antenna height: $3 \mathrm{~m}$, output power ERP $42.210 \mathrm{dBW}$, equivalent $16.6 \mathrm{~kW}$ ) with the TV station located at Saigon Center, 65 Le Loi, District 1, Ho Chi Minh City and ii) VTV1 (channel 21 with frequency $474 \mathrm{MHz}$, transmit antenna height: $180 \mathrm{~m}$, receive antenna height: $3 \mathrm{~m}$, output power ERP 56.840 $\mathrm{dBW}$, equivalent $483 \mathrm{~kW}$ ). The TV transmitter is located at Thuan An - Binh Duong province. The measurement is based on the Promax Prolink-4/4C Premium ${ }^{4}$, which is able to demodulate Pal D/K and DVB-T/T2 signals.

It is noted that the ITU-R 1546.1 results are obtained after the correction factor with antenna height $3 \mathrm{~m}$ and environment type. The correction factor is a function of the receive antenna height, operating frequency and receiver surrounding environment. For channel 62 (802 $\mathrm{MHz}$ ), the correction factor are -11.0881 for free-space, -23.9098 for suburban and -27.3896 for urban, respectively. Similarly, the corresponding factors of channel 21 $(474 \mathrm{MHz})$ for free space, suburban and urban are $-10.3477,-21.6135$ and -25.0889 , respectively. The antenna height for both two cases is $3 \mathrm{~m}$.

Figure 2 shows that the ITU-R 1546.1 model achieves the RMSE of 10.38 with the correction factor, see Table IV. It is shown that the ITU-R 1546 root mean square error (RMSE) is lower than that of the $F(X, Y)$, i.e. 23.64. Since the distance is in between 10 and $20 \mathrm{~km}$, it can be seen that the measured result is not agreement with the ITU-R 1546. It can be explained by making use the hidden-node effect of wireless channels. At this point, we can conclude that the ITU-R 1546 is better than the $\mathrm{F}(\mathrm{X}, \mathrm{Y})$. As a result, we choose the ITU-R 1546.1 as the channel model for our analysis in the next section.

\section{Channel Propagation Model for TVWS Devices}

We now consider the channel propagation model for WSDs. It should be noted that fixed devices are permitted to transmit up to a $4 \mathrm{~W}$ equivalent of effective isotropic radiated power (EIRP), with $1 \mathrm{~W}$ output power and a $6 \mathrm{dBi}$ gain antenna per channel. For personal/portable devices, the maximum transmit power is $100 \mathrm{~mW}$ equivalent EIRP with no antenna gain. In this section, we consider three models for TVWS devices including free space path loss model, two ray ground reflection model and Okumura-Hata model.

\footnotetext{
${ }^{4}$ http:/ / www.promax.es
}

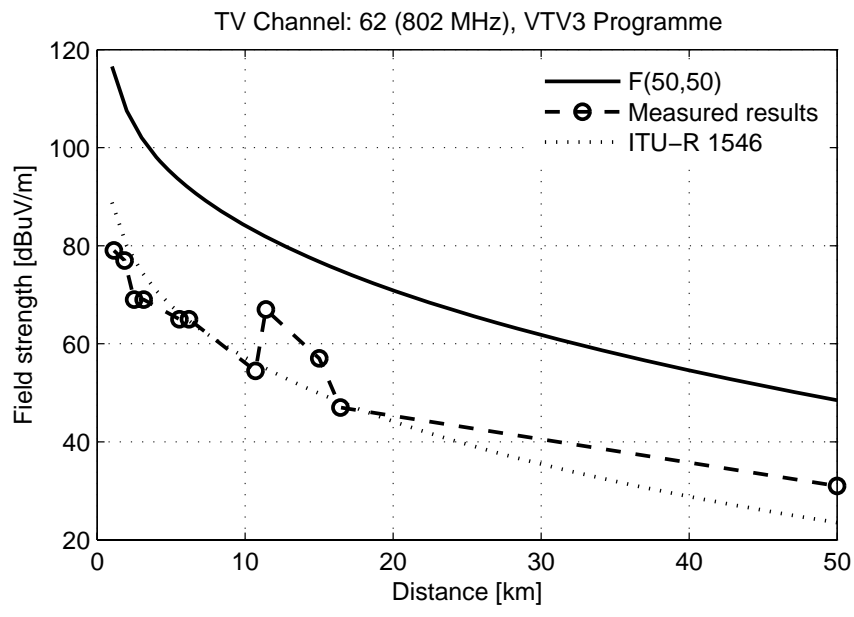

Figure 2. Measurement results vs. Theory results

\subsection{Free Space Path Loss Model}

Consider a signal transmitted though free space to a TV receiver located at distance $d$ from the CR transmitter. Given that there are no obstructions in between and the signal propagates along a straight line, we have [19]

$$
\frac{P_{r}}{P_{t}}=\left(\frac{\sqrt{G_{t} G_{r}} \lambda}{4 \pi d}\right)^{2},
$$

where $P_{t}$ is the transmit power and $P_{r}$ is the receive power. Such a model is called as line-of-sight (LOS) channel. Furthermore, $G_{t}$ and $G_{r}$ denote the antenna gains of the transmitter and the receiver, respectively, and $\lambda$ is the wavelength.

\subsection{Two Ray Ground Reflection Model}

The two-ray model is a commonly used propagation model since it accounts for a ground-reflected path between transmitter and receiver in addition to the LOS component. The two-ray model has been shown to produce more accurate path-loss estimates at long distances than the Friis free space equation since antenna height differences at transmitter and receiver is taken into account. For a given distance $d$ from the transmitter, the received power is estimated by [19]

$$
P_{r}=\left\{\begin{array}{c}
\left(\frac{\sqrt{G_{t} G_{r}} h_{t} h_{r}}{d^{2}}\right)^{2} P_{t}, \quad d>d_{c} \\
\left(\frac{\sqrt{G_{t} G_{r}} \lambda}{4 \pi d}\right)^{2} P_{t}, \quad d \leq d_{c}
\end{array},\right.
$$

where $h_{t}$ and $h_{r}$ are the heights of the transmit and receive antennas respectively. In (9), $d_{c}$ denotes the cross-over distance given by

$$
d_{c}=4 h_{t} h_{r} / \lambda .
$$

\subsection{Okumura-Hata Model}

Okumura-Hata model is one of the most widely used models for signal coverage prediction in urban areas [20,21]. This model is applicable for distances of $1-100 \mathrm{~km}$ and for frequencies in the range of 150-1920 $\mathrm{MHz}$. Base station antenna heights, which are valid for 
Table IV

Comparison of ITU 1546.1, F(50,50) AND the Measurement Results

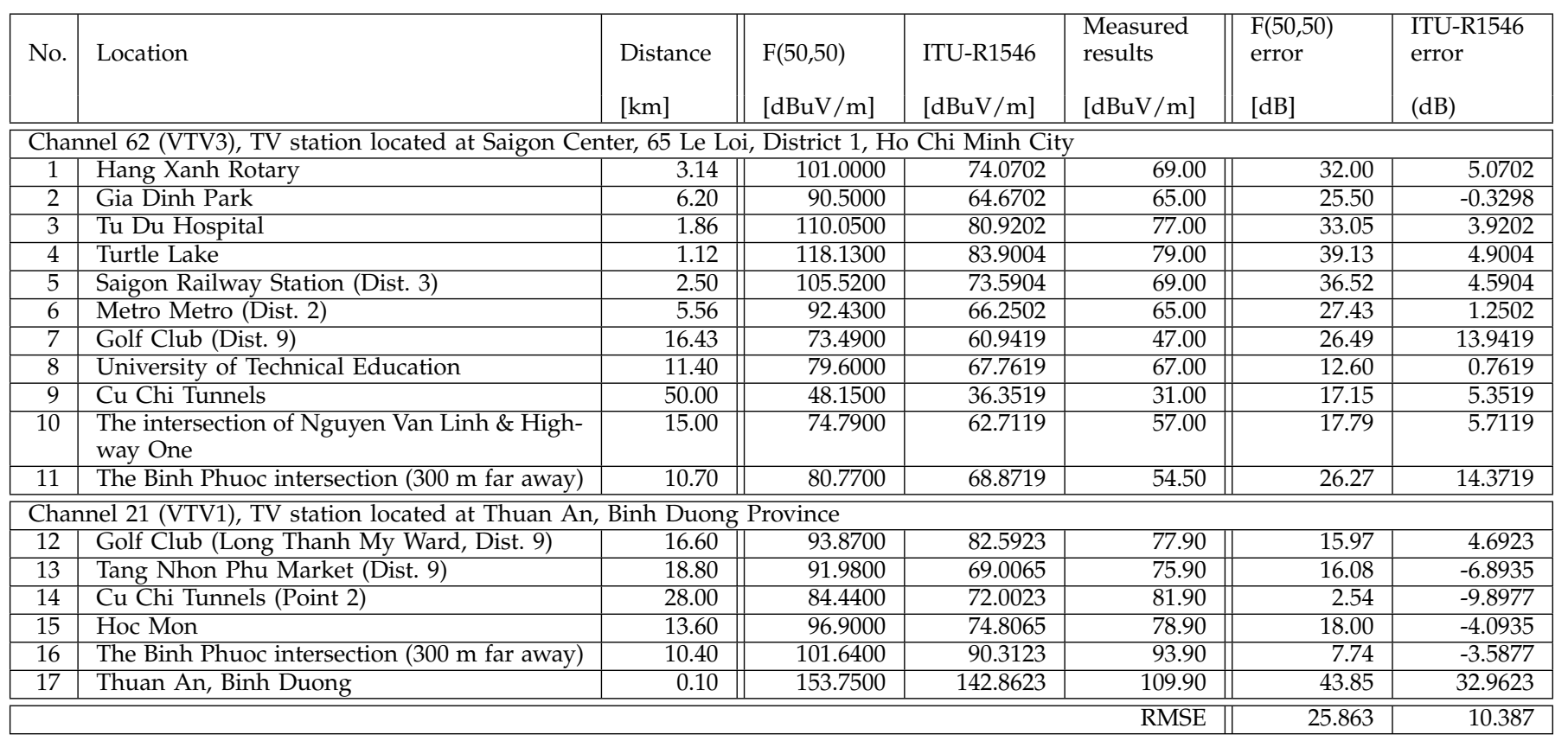

the model, are from 30 to $1000 \mathrm{~m}$. The path-loss in $\mathrm{dB}$, $P L=10 \log _{10} P_{t} / P_{r}$, is written as

$$
\begin{aligned}
\mathrm{PL}= & 69.55+26.16 \log f-13.82 \log h_{t}-a\left(h_{r}\right) \\
& +\left[44.9-6.55 \log h_{t}\right] \log d+C .
\end{aligned}
$$

In (11), operating frequency $f$ is given in $\mathrm{MHz}$ and distance $d$ in $\mathrm{km}$. Furthermore, function $a\left(h_{r}\right)$ and factor $C$, which depend on the environment, are given in [22, Appendix 7.A].

\subsection{Comparison with Measurement Results}

Cognitive devices for field measurement, called RuralConnect, consists of a base station (BS) and a fixed customer premises equipment (CPE). All softwaredefined radio designed cognitive devices are provided by Carlsonwireless ${ }^{5}$, operating on UHF band (470-786 $\mathrm{MHz}$ ) with bandwith of $8 \mathrm{MHz}$. The Federal Communications Commission (FCC) has certified the RuralConnect TVWS radio system for use with TV white spaces database. RuralConnect delivers extended coverage, non-line-of-sight (NLOS) broadband connectivity by transmitting over TV white space (TVWS) frequencies, which offer superior signal propagation characteristics. The RF transmit power for BS and for CPE are 26 $\mathrm{dBm}$ and $25 \mathrm{dBm}$, respectively. The maximum transmit power equivalent in ERP is $36 \mathrm{dBm}$ for both BS and CPE. Modulation settings are provided in Table V. Table VI provides configuration for RuralConnect BS and CPE.

In Hanoi, the measurement is performed in 10 different positions of CPE on July 2013. The TV channel under investigation is $43(650 \mathrm{MHz})$. The BS is located at the national television broadcaster of Vietnam (43 Nguyen Chi Thanh Street, Hanoi), or VTV, with

\footnotetext{
${ }^{5}$ http:/ / www.carlsonwireless.com/
}

Table V

Modulation AND Tx Threshold.

\begin{tabular}{|l|c|}
\hline Modulation & Rx Threshold [dBm] \\
\hline \hline QPSK1/2 & -88 \\
\hline QPSK3/4 & -86 \\
\hline 16QAM1/2 & -85 \\
\hline 16QAM3/4 & -83 \\
\hline 16QAM & -81 \\
\hline
\end{tabular}

Table VI

Antenna Configuration for Measurement

\begin{tabular}{|l||c|c|}
\hline Antenna & Base Station & CPE \\
\hline \hline Type & Omni & Sector \\
\hline Beamwidth & 360 & 90 \\
\hline Polarization & $\mathrm{V}$ & V or H \\
\hline Frequency Range in $\mathrm{MHz}$ & $470-786$ & $470-786$ \\
\hline Gain in dBi & 6 & 10 \\
\hline Impedence $(\Omega)$ & 75 & 75 \\
\hline
\end{tabular}

transmit antenna of $35 \mathrm{~m}$. The transmission effective radiated power (ERP) is $36 \mathrm{dBm}$ (4W EIRP). In Ho Chi Minh city, we consider channel 45 with frequency 666 $\mathrm{MHz}$ and the measurement site is at District 9, whose locations are shown in Figure 3. The BS is placed at the transmission site of the voice of HCM City people (Man Thien Street, District 9).

It is observed from Figure 4 that the OkumuraHata model outperforms the free space model, which, in turns, outperforms the two ray ground reflection model. In the next section, we will adopt the OkumuraHata model as the channel model between fixed CR devices.

\section{The Proposed Algorithm}

Having chosen two propagation models, i.e. ITU 1564.1 and Okumura Hata, we are now in a position to pro- 
Table VII

Comparison of Free Space, Two Ray, OKumura-Hata and the Measurement Result

\begin{tabular}{|c|c|c|c|c|c|c|c|}
\hline Locations & $\begin{array}{l}\text { Measurement } \\
\text { results } \\
\quad(\mathrm{dBm})\end{array}$ & $\begin{array}{l}\text { Free Space } \\
(\mathrm{dBm})\end{array}$ & $\begin{array}{c}\text { Two Ray } \\
\text { (dBm) }\end{array}$ & $\begin{array}{l}\text { Okumura- } \\
\text { Hata } \\
\qquad(\mathrm{dBm})\end{array}$ & $\begin{array}{l}\text { Free Space } \\
\text { error } \\
\qquad(\mathrm{dBm})\end{array}$ & $\begin{array}{c}\text { Two-Ray error } \\
(\mathrm{dBm})\end{array}$ & $\begin{array}{r}\text { Okumura- } \\
\text { Hata error } \\
(\mathrm{dBm})\end{array}$ \\
\hline CPE1 & -66.00 & -26.77 & 0.75 & -42.12 & 39.23 & 66.75 & 23.88 \\
\hline CPE2 & -83.00 & -38.25 & -22.2 & -62.09 & 44.75 & 60.8 & 20.91 \\
\hline CPE3 & -83.00 & -43.52 & -32.73 & -71.24 & 39.48 & 50.27 & 11.76 \\
\hline CPE4 & -63.00 & -32.44 & -6.64 & -37.86 & 30.56 & 56.36 & 25.14 \\
\hline CPE5 & -65.00 & -30.86 & -3.47 & -35.11 & 34.14 & 61.53 & 29.89 \\
\hline CPE6 & -75.00 & -36.88 & -15.51 & -45.58 & 38.12 & 59.49 & 29.42 \\
\hline CPE7 & -81.70 & -40.96 & -23.67 & -52.68 & 40.74 & 58.03 & 29.02 \\
\hline CPE8 & -84.00 & -46.42 & -34.5 & -62.17 & 37.58 & 49.5 & 21.83 \\
\hline & & & & RMSE & 38.28 & 58.09 & 24.63 \\
\hline
\end{tabular}

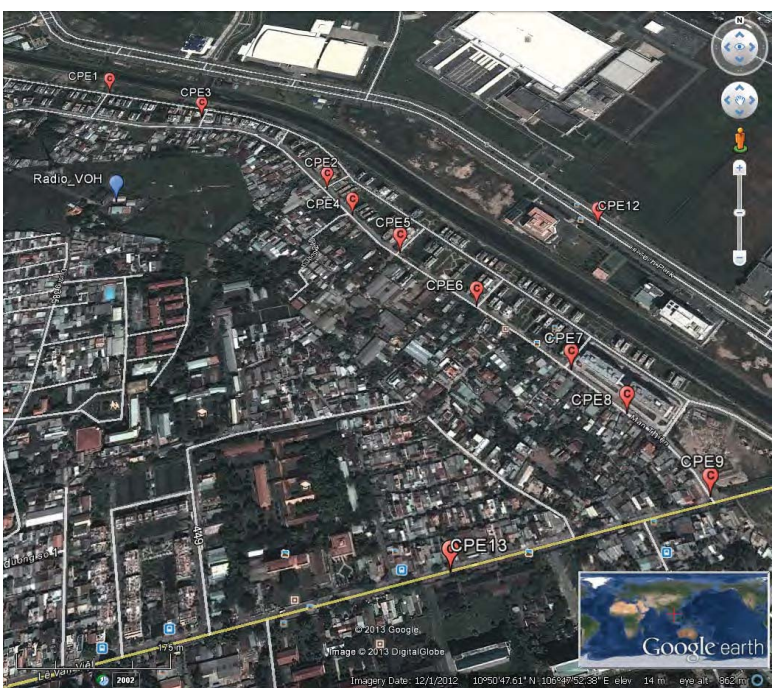

Figure 3. CPE locations at the transmit site of the voice of Ho Chi Minh city people (Man Thien Street, District 9).

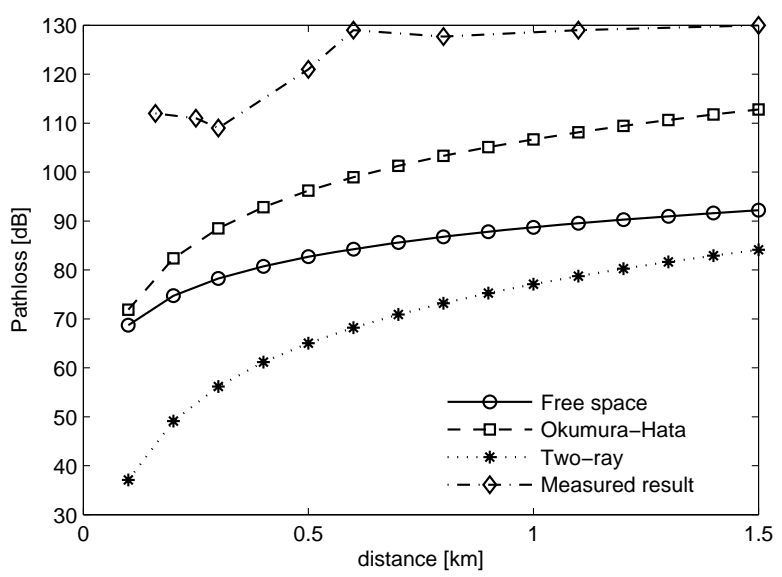

Figure 4. Comparision of free space, two ray, Okumura-Hata and the measured results.

pose a new algorithm to estimate regional TV whitespace usability. A good starting point is the analysis of the maximum permitted transmit power of secondary users. In particular, WRAN CPEs and BSs will not be allowed to operate on the same channel or on the first adjacent channels of a TV operation within the TV protected contour. However, communications on co-channels or adjacent channels outside this protected contour are possible as long as they are located at sufficient keep-out distances beyond this protected contour. WRAN CPEs or BSs located outside the TV protected contour but within the range of the keepout distance must adjust their maximum EIRP accordingly to protect TV operation. WRAN CPEs and BSs operating on alternate channels (i.e. $N \pm 2, N \pm 3, \ldots$ ) may be located inside the TV protected contour as long as they meet the maximum transmit EIRP limits defined by the EIRP profile. This EIRP profile defines the maximum EIRP limit that a WRAN CPE or BS will not exceed a reference minimum distance of $100 \mathrm{~m}$ from a TV receiver, as a function of the channel separation between the TV operation and the WRAN operation, in order to avoid causing harmful interference.

The flow chart of the proposed algorithm is given in Figure 5, where the reference minimum distance, typically $d_{\min }=100 \mathrm{~m}$. If $d<d_{\text {min }}$, the channel will be advance to the black list. Otherwise, it will be added to the white list. It is noted that due to frequency reuse, some of channels on the white list will also be on the black list and vice versa. As a result, the proposed algorithm will make the comparison and channels appearing on both lists will be eliminated. The TV database consists of TV VHF/UHF (Pal D/K, DVBT, DVB-T2) broadcasters in Vietnam, which is provided by Vietnam Authority of Radio Frequency Management [23]. The data-set includes coordinates, transmit power (ERP), antenna gain, and active channels. Note that WSDs from Carlson only work with UHF band, i.e. Carlson's Rural Connect only active on channel 21-51. To reduce the computation complexity in building TV database, we limit our database on UHF band (band $\mathrm{IV} / \mathrm{V})$, i.e. $470-806 \mathrm{MHz}$. This band is also planned for digital TV in Vietnam.

Given the coordinate of a TV broadcaster and a CR transmitter, we have

$$
d+R_{\mathrm{TV}}=D
$$

where $d$ is the distance away from the edges of the TV transmitter. $R_{\mathrm{TV}}$ is the TV coverage radius determined by the ITU-R 1546.1 model and $D$ is the distance between two points over the Earth from their longitudes and latitudes. $D$ is derived from the Haversine formula, e.g. [24]

$$
D=2 R \arcsin \left[\min \left(1, \sqrt{\sin ^{2} \phi+\cos \phi_{1} \cos \phi_{2} \sin ^{2} \lambda}\right)\right],
$$




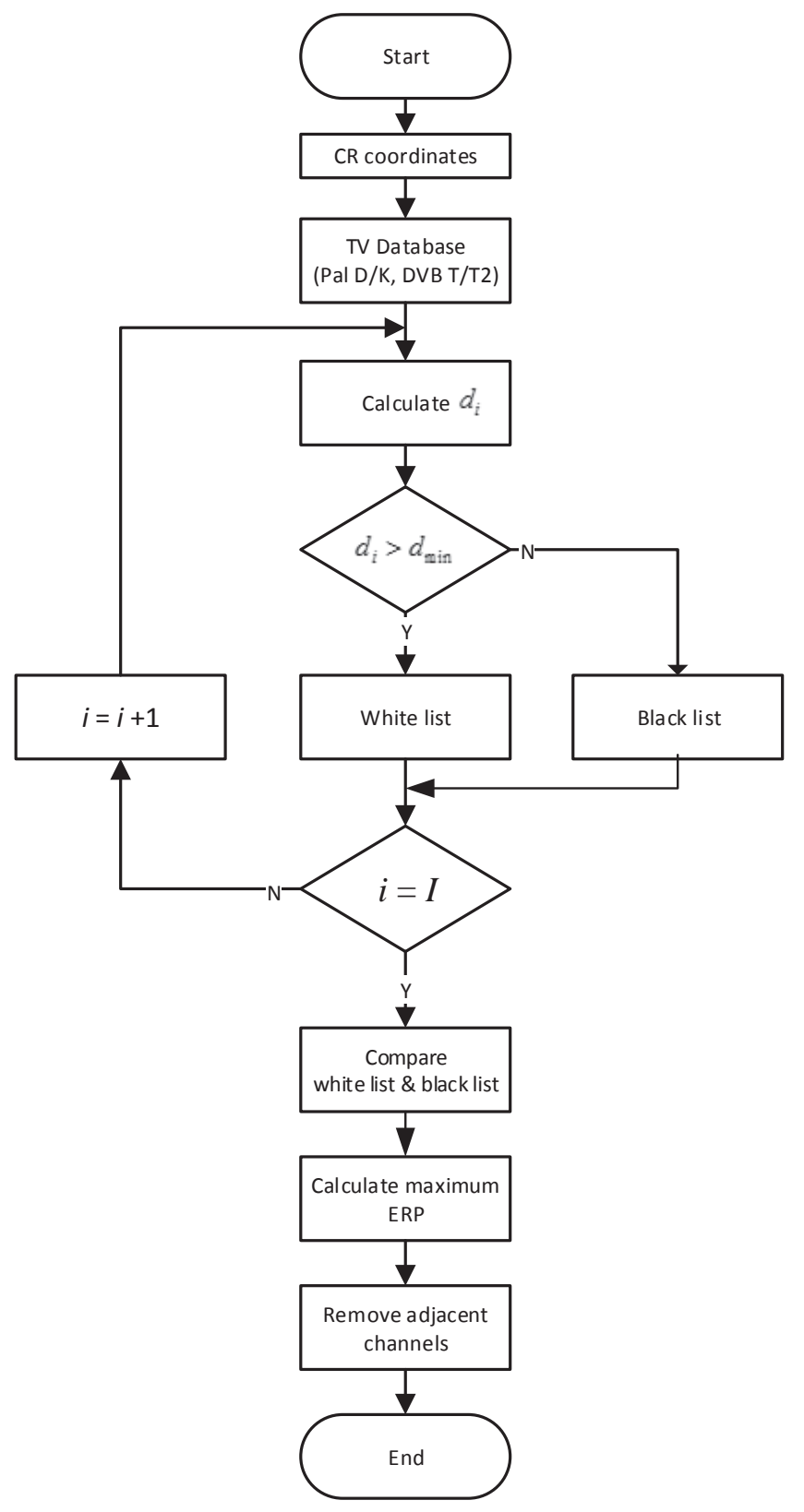

Figure 5. The flowchart of the proposed algorithm, I denotes the number of TV broadcast stations.

where $R$ is the radius of the Earth, about 6,371 kilometers. Furthermore, $\phi$ and $\lambda$ are respectively defined as

$$
\begin{aligned}
& \phi=\frac{\phi_{2}-\phi_{1}}{2}, \\
& \lambda=\frac{\lambda_{2}-\lambda_{1}}{2},
\end{aligned}
$$

where $\phi_{k}$ and $\lambda_{k}$ with $k \in\{1,2\}$ respectively denote latitude and longitude of point $k$.

Starting from the desired-to-undesired signal ratio ( $\mathrm{D} / \mathrm{U}$ ratio) and adopting that the interference is a WRAN signal, the maximum undesired field strength at the noise-limited contour, $E_{C R_{\max }}$, is given by [25]

$$
\begin{aligned}
E_{C R_{\max }}= & \min \left[E_{T V}-(D / U)_{c c}+F / B,\right. \\
& \left.E_{T V}-(D / U)_{a c}+F / B\right],
\end{aligned}
$$

where $E_{T V}$ is the desired field strength, i.e. $47 \mathrm{dBu}$ for
DVB-T with IV/V UHF band), which is the signal TV that needs to be protected. $F / B$ is a front-to-back radio for the TV receive antenna, i.e. $F / B=17 \mathrm{~dB}[25, \mathrm{Eq}$. (14)]. $D / U_{c c}$ is the co-channel $\mathrm{D} / \mathrm{U}$ ratio and $D / U_{a c}=$ $-9 \mathrm{~dB}$ is the adjacent $\mathrm{D} / \mathrm{U}$ ratio $[26$, p. 6]. Placing all together into (16), we have

$$
\begin{aligned}
E_{C R_{\max }} & =\min [47-40+17,47-(-9)+17] \\
& =24 \mathrm{dBu} .
\end{aligned}
$$

Converting $E_{C R_{\max }}$ from $\mathrm{dBu}$ to $\mathrm{dBm}$ gives [27]

$$
E_{C R_{\max }}[\mathrm{dBm}]=E_{C R_{\max }}[\mathrm{dBu}]-20 \log _{10} f_{\mathrm{MHz}}+G_{\mathrm{dBi}}-77.2 \text {, }
$$

where $G_{d B i}$ denotes antenna gain in $\mathrm{dBi}$.

Having the maximum undesired field strength at the noise limited contour, we can calculate the maximum undesired field strength at WRAN BS and CPE (also called WSDs) as

$$
\begin{aligned}
\operatorname{EIRP}_{W S D}[\mathrm{dBm}]= & E_{C R_{\max }}[\mathrm{dBm}]+69.55+26.16 \log f \\
& -13.82 \log h_{t}-a\left(h_{r}\right) \\
& +\left[44.9-6.55 \log h_{t}\right] \log d+C,
\end{aligned}
$$

where $a\left(h_{r}\right)$ and $C$ are given in (11).

\section{Numerical Results}

The purpose of this section is to confirm the advantage of the proposed algorithm. As a reference, the results of the keep away algorithm [10] and the algorithm in [11] on the same settings are also illustrated. The Okumura-Hata channel model is used as the channel model for calculating the maximum transmit power for CR devices. The method for determining vacant channels is connecting with TV database. We limit our study to 22 provinces on the South of Vietnam with UHF channels from channel 21 to channel 62. Other setting parameters are shown in Table VIII.

Table VIII

Measurement Setting Parameters

\begin{tabular}{|l|c|}
\hline Parameters & Remarks \\
\hline Cochannel D/U & $40 \mathrm{~dB}$ \\
\hline Adjacent D/U & $-9 \mathrm{~dB}$ \\
\hline Desired field strength (Band IV/V UHF) & $47 \mathrm{dBu}$ \\
\hline Channel model for TV stations & ITU-R 1546 \\
\hline Channel model for WSDs & Okumura-Hata \\
\hline CR output power (EIRP) & $4 \mathrm{~W}(36 \mathrm{dBm}$ EIRP) \\
\hline CR antenna height & $30 \mathrm{~m}$ \\
\hline TV receive antenna height & $10 \mathrm{~m}$ \\
\hline
\end{tabular}

In Table IX, we can see that the proposed algorithm and the algorithm in [11] give the same number of TVWS channels. Both of them outperform the keepaway one in [10]. In all channels under consideration, the maximum transmitted EIRPs from the algorithm in [11] is smaller than our proposed algorithm. In addition, the proposed one not only provides better coverage but also assures no harmful interference to licensed services. It can be explained by making use the fact that the Okumura-Hata model can predict path loss link budget better than the two-ray model. 
Table IX

Number of TVWS Channels in Tien Giang Province (USERcode = TNGG0026).

\begin{tabular}{|c||c|c|c|c|}
\hline & & The keep-away algorithm[10] & The power adaptation algorithm [11] & The proposed algorithm \\
\hline No. & Channel & \multicolumn{3}{|c|}{ The maximum power EIRP [dBm] } \\
\hline \hline 1 & 21 & 36 & 36.00 & 36.00 \\
\hline 2 & 22 & 36 & 36.00 & 36.00 \\
\hline 3 & 23 & 36 & 36.00 & 36.00 \\
\hline 4 & 24 & 36 & 36.00 & 36.00 \\
\hline 5 & 25 & - & 30.17 & 36.03 \\
\hline 6 & 27 & 36 & 36.00 & 36.00 \\
\hline 7 & 28 & - & 36.00 & 36.00 \\
\hline 8 & 29 & 36 & 36.00 & 36.00 \\
\hline 9 & 30 & 36 & 36.00 & 36.00 \\
\hline 10 & 31 & 36 & 36.00 & 36.00 \\
\hline 11 & 32 & 36 & 36.00 & 36.00 \\
\hline 12 & 33 & 36 & 36.00 & 34.22 \\
\hline 13 & 34 & 36 & 36.00 & 36.00 \\
\hline 14 & 35 & - & 30.43 & 10.99 \\
\hline 15 & 36 & - & 36.00 & 36.00 \\
\hline 16 & 38 & - & 3.82 & 32.62 \\
\hline 17 & 41 & - & 36.00 & 36.00 \\
\hline 18 & 42 & - & 28.10 & 36.00 \\
\hline 19 & 43 & 36 & 36.00 & 36.00 \\
\hline 20 & 44 & 36 & 36.00 & 36.00 \\
\hline 21 & 45 & 36 & 36.00 & 36.00 \\
\hline 22 & 46 & 36 & 36.00 & 32.44 \\
\hline 23 & 47 & 36 & 36.00 & 36.00 \\
\hline 24 & 48 & - & 27.47 & 36.00 \\
\hline 25 & 49 & 36 & 36.00 & 36.00 \\
\hline 26 & 50 & 36 & 36.00 & 32.09 \\
\hline 27 & 53 & - & 36.00 & 32.04 \\
\hline 28 & 60 & - & 26.34 & \\
\hline 29 & 61 & - & 1.54 & \\
\hline 30 & 62 & & & \\
\hline & & - & 26.17 & \\
\hline
\end{tabular}

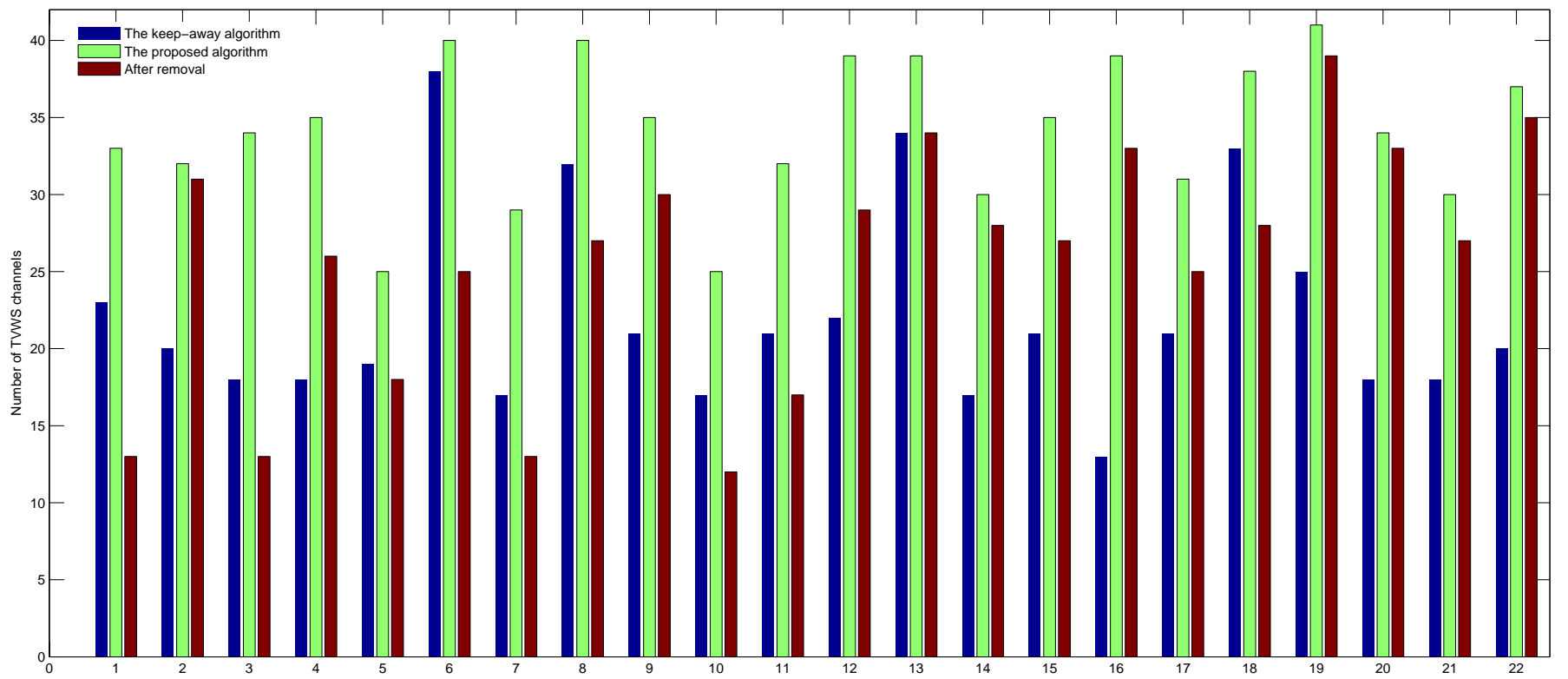

Figure 6. Number of TVWS channels for CR devices at 22 different locations: with and without adjacent channels.

In Figure 6 and Table $X$, we study the number of TVWS provided from the keep-away algorithm and the proposed algorithm. In particular, over 22 provinces, the average number of TVWS channels of the keep-away algorithm and the proposed algorithm are 22.09091 and 34.2272. If adjacent channels are not used, the number of TVWS channels are reduced to 25.59. In addition, we can see the proposed algorithm can increase to $54.93 \%$ the average number of TVWS channels. 
Table X

Comparison Number of TVWS Channels for CR Devices at 22 Different Locations: with and without Adjacent Channels.

\begin{tabular}{|c|c|c|c|c|c|c|}
\hline \multirow[b]{3}{*}{ No. } & \multirow[b]{3}{*}{ Province } & \multirow[b]{3}{*}{ Usercode } & \multirow{3}{*}{$\begin{array}{l}\text { Coordinates } \\
\text { (Lat; Long) }\end{array}$} & \multicolumn{3}{|c|}{ Number of TVWS channels } \\
\hline & & & & \multirow{2}{*}{$\begin{array}{l}\text { The keep-away } \\
\text { algorithm [10] }\end{array}$} & \multicolumn{2}{|c|}{ The proposed algorithm } \\
\hline & & & & & $\begin{array}{l}\text { With adjacent } \\
\text { channels }\end{array}$ & $\begin{array}{l}\text { Without adjacent } \\
\text { channels }\end{array}$ \\
\hline 1 & Binh Thuan & BHTN0001 & $11.186550000 ; 107.559894400$ & 23 & 33 & 13 \\
\hline 2 & Long An & LGAN0026 & $10.534552780 ; 105.784711100$ & 20 & 32 & 31 \\
\hline 3 & Ba Ria & BRVT0001 & $10.490277780 ; 107.184250000$ & 18 & 34 & 13 \\
\hline 4 & Ben Tre & BNTE0634 & $10.238333330 ; 106.380555600$ & 18 & 35 & 26 \\
\hline 5 & Binh Duong & BHDG0758 & $10.972544440 ; 106.673313900$ & 19 & 25 & 18 \\
\hline 6 & Ca Mau & CAMA0086 & $9.137511110 ; 107.788055600$ & 38 & 40 & 25 \\
\hline 7 & Can Tho & CNTO0758 & $10.038319440 ; 105.788133300$ & 17 & 29 & 13 \\
\hline 8 & Lam Dong & LMDG0003 & $11.760911110 ; 108.494600000$ & 32 & 40 & 27 \\
\hline 9 & Vinh Long & VHLG0086 & $10.112222220 ; 104.888888900$ & 21 & 35 & 30 \\
\hline 10 & Ho Chi Minh City & HCMC0634 & $10.773580560 ; 106.700944400$ & 17 & 25 & 12 \\
\hline 11 & An Giang & ANGG0007 & $10.396111000 ; 105.421666700$ & 21 & 32 & 17 \\
\hline 12 & Bac Lieu & BCLU0011 & $9.296388889 ; 105.689722200$ & 22 & 39 & 29 \\
\hline 13 & Binh Phuoc & BHPC0022 & $11.824166670 ; 107.005555600$ & 34 & 39 & 34 \\
\hline 14 & Dong Thap & DGTP0001 & $10.463694440 ; 105.635819400$ & 17 & 30 & 28 \\
\hline 15 & Kien Giang & KNGG0634 & $10.111666670 ; 104.888333300$ & 21 & 35 & 27 \\
\hline 16 & Dong Nai & DGNI0113 & $10.940000000 ; 107.375833300$ & 13 & 39 & 33 \\
\hline 17 & Soc Trang & SCTG0758 & $9.590130556 ; 105.976605600$ & 21 & 31 & 25 \\
\hline 18 & Ninh Thuan & NHTN0086 & $11.576800000 ; 108.978200000$ & 33 & 38 & 28 \\
\hline 19 & Tay Ninh & TANI0634 & $11.368888890 ; 106.165555600$ & 25 & 41 & 39 \\
\hline 20 & Hau Giang & HUGG0014 & $9.791111111 ; 105.472777800$ & 18 & 34 & 33 \\
\hline 21 & Tien Giang & TNGG0758 & $10.353063890 ; 106.358344400$ & 18 & 30 & 27 \\
\hline 22 & Tra Vinh & TAVH0017 & $9.949166667 ; 106.346388900$ & 20 & 37 & 35 \\
\hline
\end{tabular}

\section{Conclusion}

This paper has proposed a new algorithm to calculate TVWS channels for cognitive radio based on the ITU 1546.1 and the Okumura-Hata model. The proposed algorithm is verified on the data of 22 provinces in the South of Vietnam. Numerical results show that the power adaptation algorithm always provides better the number of TVWS channels than the keep-away algorithm. It should be noted that the proposed algorithm can be straightforwardly extended for the whole Vietnam if the TV database is available. In this paper, we only consider fixed CR devices with the OkumuraHata model. However, the Okumura-Hata model is not recommended for $\mathrm{CR}$ devices with antenna height lower than $30 \mathrm{~m}$, e.g. portable devices. Our future study is for portable WSDs.

\section{ACKNOWLeDgments}

This work of the second author is supported by Project 272/HD-SKHCN granted by Ho Chi Minh City Department of Science and Technology.

\section{REFERENCES}

[1] W. Webb, "On using white space spectrum," IEEE Communications Magazine, vol. 50, no. 8, pp. 145-151, Aug. 2012.

[2] M. Wellens, J. Wu, and P. Mahonen, "Evaluation of spectrum occupancy in indoor and outdoor scenario in the context of cognitive radio," in Proc. 2nd Int. Conf. Cognitive Radio Oriented Wireless Networks and Communications (CrownCom) 2007, 2007, pp. 420-427.

[3] M. Lpez Bentez and F. Casadevall, "Methodological aspects of spectrum occupancy evaluation in the context of cognitive radio," European Transactions on Telecommunications, 2009.

[4] S. Pagadarai, A. M. Wyglinski, and R. Vuyyuru, "Characterization of vacant UHF TV channels for vehicular dynamic spectrum access," in 2009 IEEE Vehicular Networking Conference (VNC), 2009, pp. 1-8.

[5] V. N. Q. Bao, L. Q. Cuong, L. Q. Phu, T. D. Thuan, L. M. Trung, and N. T. Quy, "Spectrum survey in vietnam: Occupancy measurements and analysis for cognitive radio applications," in Proc. The 2011 International Conference on Advanced Technologies for Communications, 2011, pp. 135143.

[6] S. Chin-Sean, G. P. Villardi, M. A. Rahman, T. Baykas, T. Ha Nguyen, L. Zhou, S. Chen, Y. Alemseged, W. Junyi, S. Chunyi, P. Chang-woo, S. Filin, and H. Harada, “Cognitive communication in TV white spaces: An overview of regulations, standards, and technology [accepted from open call]," IEEE Communications Magazine, vol. 51, no. 7, pp. 138-145, Jul. 2013.

[7] A. Goldsmith, S. A. Jafar, I. Maric, and S. Srinivasa, "Breaking spectrum gridlock with cognitive radios: An information theoretic perspective," Proceedings of the IEEE, vol. 97, no. 5, pp. 894-914, May 2009.

[8] S. Y. Lee, M. K. Kwon, and S. H. Lee, "Transmit power control scheme for TV white space wireless system," in Proc. 13th International Conference on Advanced Communication Technology (ICACT), 2011, pp. 1025-1029.

[9] V. Petrini and H. R. Karimi, "TV white space databases: Algorithms for the calculation of maximum permitted radiated power levels," in Proc. 2012 IEEE International Symposium on Dynamic Spectrum Access Networks (DYSPAN), 2012, pp. 552-560.

[10] L. Xiuqin and F. Yang, "A survey on the available digital TV bands for cognitive radio operations," in Proc. Fourth International Conference on Communications and Networking in China, 2009 (ChinaCOM 2009), 2009, pp. 1-7.

[11] N. Wang, Y. Gao, Y. Chen, E. Bodanese, and L. Cuthbert, "Performance evaluation of power control algorithm for TV white space resource in UK," in Proc. 2012 7th International ICST Conference on Communications and Networking in China (CHINACOM 2012), 2012, pp. 733-736.

[12] IEEE, "IEEE 802.22 working group on wireless regional 
area networks," 2011. [Online]. Available: http:// grouper.ieee.org/groups/802/22

[13] R. A. O'Connor, "Understanding television's grade A and grade B service contours," IEEE Transactions on Broadcasting, vol. 47, no. 3, pp. 309-314, Sep. 2001.

[14] ITU, “Recommendation ITU-R BT.417-5: Minimum field strengths for which protection may be sought in planning an analogue terrestrial television service," pp. 1-2, 2002. [Online]. Available: https://www.itu.int/rec/ R-REC-BT.417-5-200210-I/en

[15] ECC, "ECC report 159: Technical and operational requirements for the possible operation of cognitive radio systems in the white spaces of the frequency band 470-790 MHz," Report, 2011. [Online]. Available: http://www.erodocdb.dk/docs/ doc98/official/pdf/ECCRep159.pdf

[16] - "Annex to ECC report 4: Criteria for planning DVB-T," Report, 2007. [Online]. Available: http://www.erodocdb.dk/Docs/doc98/official/ pdf/ECCRep004 Technical Annex.pdf

[17] ITU, "P.1546: Method for point-to-area predictions for terrestrial services in the frequency range 30 $\mathrm{MHz}$ to $3000 \mathrm{MHz}, "$ 2013. [Online]. Available: https: //www.itu.int/rec/R-REC-P.1546/en

[18] J. Damelin, W. A. Daniel, H. Fine, and G. V. Waldo, Development of VHF and UHF propagation curves for $T V$ and FM broadcasting. Federal Communications Commission, 1966. [Online]. Available: www.fcc.gov/ oet/info/documents/reports/R-6602.pdf

[19] A. Goldsmith, Wireless communications. Cambridge ; New York: Cambridge University Press, 2005.

[20] Y. Okumura, E. Ohmori, T. Kawano, and K. Fukuda, "Field strength and its variability in VHF and UHF landmobile radio service," Rev. Elec. Commun. Lab, vol. 16, no. 9, pp. 825-73, 1968.

[21] M. Hata, "Empirical formula for propagation loss in land mobile radio services," IEEE Transactions on Vehicular Technology, vol. 29, no. 3, pp. 317-325, Aug. 1980.

[22] A. F. Molisch, Wireless communications. John Wiley \& Sons, 2010, vol. 15.

[23] R. Vietnam, "The list of TV broadcasters." [Online]. Available: http://www.rfd.gov.vn/Noi dung/ So lieu cap+phep+PTTH/So lieu cuc moi/

[24] R. W. Sinnott, "Virtues of the Haversine," Sky and telescope, vol. 68, p. 158, Nov. 1984.

[25] I. w. group, "IEEE recommended practice for information technology - telecommunications and information exchange between systems wireless regional area networks (WRAN) - specific requirements - Part 22.2: Installation and deployment of IEEE 802.22 systems," IEEE Recommended Practice for Information Technology Telecommunications and information exchange between systems Wireless Regional Area Networks (WRAN) - Specific requirements - Part 22.2: Installation and Deployment of IEEE 802.22 Systems, 2012.

[26] ITU, "Recommendation ITU-R BT.655-7: Radiofrequency protection ratios for $\mathrm{AM}$ vestigial sideband terrestrial television systems interfered with by unwanted analogue vision signals and their associated sound signals," pp. 1-27, 2004.

[27] Semtech, "Calculating radiated power and field strength for conducted power measurements," Report, 2007. [Online]. Available: http://www.semtech.com/images/ promo/Semtech_ACS_Rad_Pwr_Field_Strength.pdf

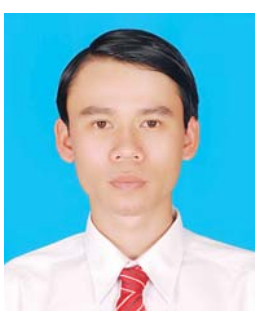

Nguyen Huu Phong received the B.E. degree in Telecommunications Engineering from University of Transport and communications Campus 2 (UTC2), Vietnam in 2006. From Nov 2012, he has been working toward the M.Eng. degree in Faculty of Telecommunications, HCMC Posts and Telecommunications Institute of Technology (PTIT). From July 2006 to June 2012, he worked as an engineer in CDMA Mobile Phone Center (S-Fone Mobile) Since July 2012, he has been a member of Research Staff in Broadcast Research and Application Center (BRAC), Vietnam Television (VTV). His research interests include the areas of mobile communication network, audio/video coding and broadcast technology

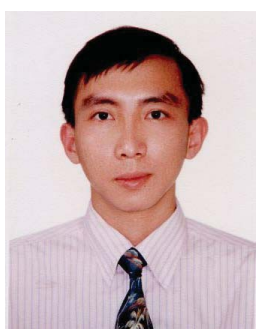

Vo Nguyen Quoc Bao was born in Nha Trang, Khanh Hoa Province, Vietnam. He received the B.E. and M.Eng. degree in electrical engineering from Ho Chi Minh City University of Technology (HCMUT), Vietnam, in 2002 and 2005, respectively, and Ph.D. degree in electrical engineering from University of Ulsan South Korea, in 2009. In 2002, he joined the Department of Electrical Engineering, Posts and Telecommunications Institute of Technology (PTIT), as a lecturer. Since February 2010, he has been with the Department of Telecommunications, PTIT, where he is currently an Assistant Professor. His major research interests are modulation and coding techniques, MIMO systems, combining techniques, cooperative communications, and cognitive radio. Dr. Bao is a member of Korea Information and Communications Society (KICS), The Institute of Electronics, Information and Communication Engineers (IEICE) and The Institute of Electrical and Electronics Engineers (IEEE). He is currently serving as the Editor of Transactions on Emerging Telecommunications Technologies (Wiley ETT). He is also a Guest Editor of EURASIP Journal on Wireless Communications and Networking, special issue on "Cooperative Cognitive Networks" and IET Communications, special issue on "Secure Physical Layer Communications".

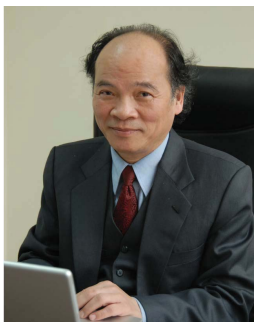

Huu-Tue Huynh was born in Hue, Viet Nam. He received the Sc.D. degree in 1972 from Laval University, Canada, where from 1969 to 2004 he was a faculty member of the Department of Electrical and Computer Engineering. In 2004, he left Laval University to become Chairman of the Department of Data Processing at The College of Technology of the Vietnam National University, Hanoi. During the period 20072011, he was the President of Bac Ha International University, Vietnam. He is now a research professor at the School of Electrical Engineering, International University, Vietnam National University, Ho Chi Minh City, Vietnam. He was an Invited Guest at The AT\&T Information Systems in Neptune, N.J. in 1984 and has been invited to give lectures at several Universities in Europe, America as well as in Asia. Professor Huynh is author and coauthor of two books and more than two hundred papers in Information Processing. He has served as Consultant to a number of Canadian Government Agencies and Industries. His research interests cover stochastic simulation techniques, information processing, fast algorithms and architectures with applications to finance and to communications 